\title{
Speckle spectro-polarimetry of solar magnetic structures
}

\author{
M. Koschinsky ${ }^{1}$, F. Kneer ${ }^{1}$, and J. Hirzberger ${ }^{1,2}$ \\ 1 Universitäts-Sternwarte Göttingen, Geismarlandstr. 11, 37083 Göttingen, Germany \\ ${ }^{2}$ Institut für Geophysik, Astrophysik und Meteorologie, Universtät Graz, Universitätsplatz 5, 8010 Graz, Austria
}

Received 19 July 2000 / Accepted 23 October 2000

\begin{abstract}
We present speckle observations of small-scale magnetic structures on the Sun. They were obtained with the "Göttingen" Fabry-Perot interferometer (FPI) in the Vacuum Tower Telescope at the Observatorio del Teide, Tenerife, from quiet and active regions close to disc center. A Stokes $V$ polarimeter was added to the FPI to measure $V$ profiles in the Fe I $6302.5 \AA$ line. The setup allows image reconstruction with speckle methods. The achieved spatial resolution in the magnetograms is $0.0^{\prime \prime} 4.0^{\prime \prime} 5$. We describe the observational technique and the data reduction. The results from small-scale magnetic flux elements in a very quiet region, in an active region with pores and abnormal granulation, and in a sunspot with its surroundings are discussed. In the quiet Sun, granular dynamics dominate the time evolution of the magnetic elements. Flux occurs in both bright intergranular points and in dark intergranular spaces. Likewise, with the present spatial resolution, no preference of magnetic flux in abnormal granulation in an active region can be found. Flux occurs in both bright (abnormal) small-scale granules and in the darker spaces in between them. The small sunspot studied has very little magnetic flux in its ambient quiet regions, especially no strong, conspicuous concentrations of returned flux, i.e. of flux with polarity opposite to that in the sunspot.
\end{abstract}

Key words. Sun: faculae, plages - Sun: magnetic fields - techniques: image processing - techniques: polarimetric

\section{Introduction}

Magnetic fields are important constituents of the solar atmosphere on all scales. Their interaction with the solar plasma causes the magnetic heating of the chromosphere and the corona and is responsible for all processes of activity from the low photosphere to the solar wind. The investigation of magnetic fields on the Sun is thus a main topic of solar research. Here, we shall concentrate on small-scale structures.

The existence of small-scale magnetic flux tubes is known since more than three decades. E.g. Beckers \& Schröter (1968) have found "magnetic knots" in the neighbourhood of sunspots as well as in quiet regions of the Sun. From the line ratio method, i.e. the use of two spectral lines which differ in their properties only by their Landé factor, Stenflo (1973) could conclude on the existence of kilo-Gauss fields with a small fill factor in the observed area. The size of the magnetic structures should be at the lower spatial resolution limit of today's telescopes or even smaller (see also the monograph by

Send offprint requests to: M. Koschinsky, e-mail: mkosch@uni-sw.gwdg.de
Stenflo 1994). Keller \& von der Lühe (1992) could show from speckle polarimetry that small magnetic structures of $0 . \prime 2$ size, or smaller, do indeed exist. A study on weak, small flux structures in quiet regions was undertaken by Stolpe \& Kneer (2000) with spectro-polarimetry and with an image scanner of the Gregory Coudé Telescope at the Observatorio del Teide/Tenerife. Likewise, Sigwarth et al. (1999) used an image scanning device and polarimeter, the Advanced Stokes Polarimeter at Sacramento Peak Observatory/New Mexico/USA, to study the properties of small magnetic elements. (The reader is referred to this work of Sigwarth et al. for much recent literature.) We also mention the efforts by, among others, Steiner et al. (1998) and Grossmann-Doerth et al. (1998) to model with numerical simulations the temporal evolution of small-scale magnetic elements.

Ground-based observations of solar finestructure suffer unavoidably from seeing effects. Recently, many efforts are undertaken worldwide to implement Adaptive Optics in solar telescopes to correct for seeing effects directly during the observations, and recent successes demonstrate its future feasibility. Meanwhile, image restoration techniques, to be applied after the observations, are well established. 


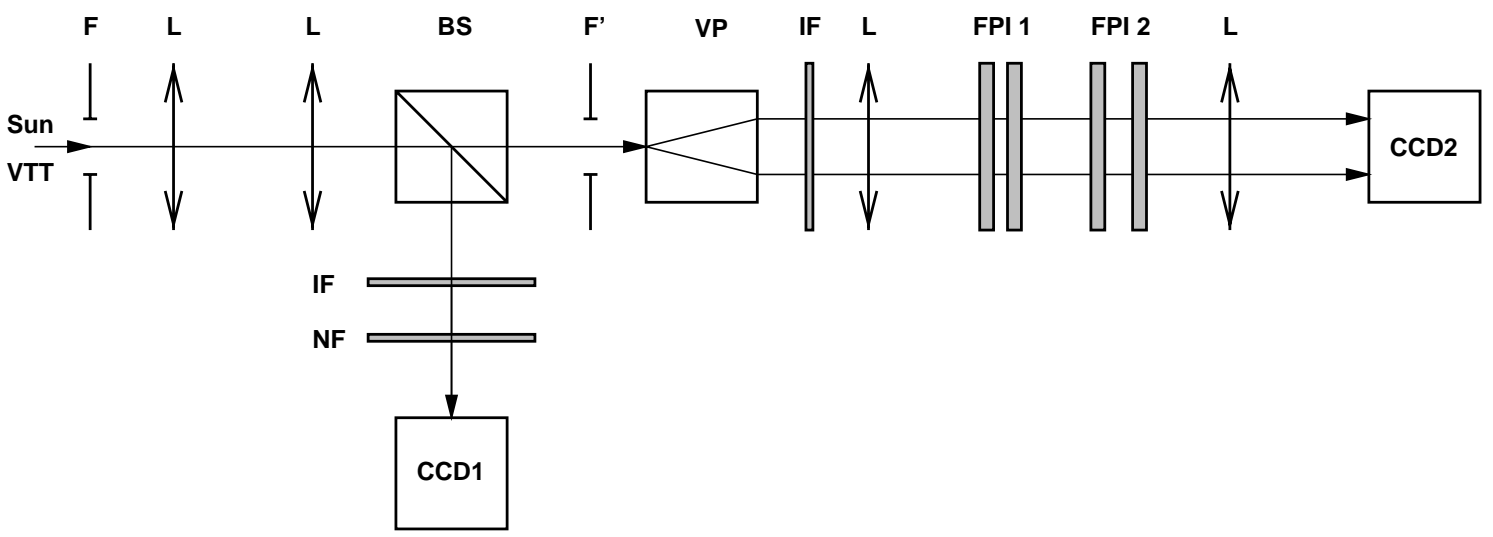

Fig. 1. Optical scheme of the two-dimensional spectrometer. F: primary focus of the VTT; $\mathrm{F}^{\prime}$ : secondary focus where the field stop is located; BS: beam splitter cube; VP: Stokes $V$ polarimeter; L: lenses; IF: interference filters; NF: neutral filter; CCD1 and CCD2: slow-scan CCDs at the foci of the broad-band and the narrow-band channels, respectively; FPI1 and FPI2: Fabry-Perot interferometers

As mentioned, Keller \& von der Lühe (1992) have used broad-band speckle polarimetry to measure the circularly polarized light components $\frac{1}{2}(I+V)$ and $\frac{1}{2}(I-V)$ at one spectral position in a magnetically sensitive line. This method cannot study the wavelength dependence of the Stokes vector across a spectral line and draw conclusions on the underlying physical processes from profile parameters such as, e.g., asymmetries, Doppler shifts, intensities, and saturation of the Stokes $V$ profiles.

To exploit the information contained in the profiles of magnetically sensitive spectral lines, we used our "Göttingen" two-dimensional Fabry-Perot spectrometer with a Stokes $V$ polarimeter. The spectrometer scans along wavelength and gives narrow-band images across spectral lines. This allows to apply image reconstruction techniques and to substantially reduce the deteriorations by seeing (see e.g. Krieg et al. 1998, 1999).

The following Sect. 2 describes the observational technique, Sect. 3 the observations for this contribution. Section 4 outlines the data analysis. In Sect. 5 we present and discuss several results from quiet and active regions. In this work, we wish to describe, along specific results, the progress in small-scale polarimetry. A detailed discussion of the rich information must be deferred to a forthcoming contribution. Section 6 concludes the paper.

\section{Observational technique}

The "Göttingen" Fabry-Perot interferometer (FPI) device is described in Bendlin et al. (1992), Bendlin (1993), and Bendlin \& Volkmer (1995). It is mounted in the Vacuum Tower Telescope (VTT) at the Observatorio del Teide/Tenerife. Here, we outline shortly the principle and the improvements which have been applied in the last years. A throughout description is given by Koschinsky (2001).

Figure 1 shows the scheme of the spectrometer, without the rather complex computer control and data aquisition systems. The Sun is imaged in the prime focus F of the VTT. From there the image is transferred to a field stop at the secondary focus $\mathrm{F}^{\prime}$ at the entrance of the spectrometer. In front of $\mathrm{F}^{\prime}$ a beam splitter cube BS takes out approximately $5 \%$ of the light to a further focus corresponding to $\mathrm{F}^{\prime}$. There broad-band images are taken with a $\mathrm{CCD}$ (CCD1) through an interference filter IF1 of 50-100 FWHM, centered at the appropriate wavelength, and a neutral filter NF. Directly after the field stop in the spectrometer a Stokes $V$ polarimeter VP was mounted for the present purposes. It was already used in earlier investigations by Volkmer (1995) and Volkmer et al. (1995). It consists of a $\lambda / 4$ retarder plate and two crossed calcites giving a separation of the left and right circularly polarized beams $\frac{1}{2}(I+V)$ and $\frac{1}{2}(I-V)$ of $4.1 \mathrm{~mm}$, adapted to the size of the CCDs. The polarimeter is followed by an appropriate interference filter IF2 of 3-10 A FWHM for order sorting of the transmission peaks of the FPIs. $\mathrm{F}^{\prime}$ is re-imaged 1:1 onto CCD2 which takes the narrow-band images. Both (slow-scan) CCDs have Thompson TH 7863 FT chips with $384 \times 286$ pixels. The image scale is $00^{\prime \prime} 1 /$ pixel and the field of view - with the polarimeter - is $166^{\prime \prime} 4 \times 27^{\prime \prime} \cdot 2$.

The two FPIs are mounted in the parallel beam near the image of the telescope's entrance pupil. This mounting is different from the telecentric mounting used in TESOS, another FPI spectrometer at the VTT (Kentischer et al. 1998) and in the IPM at THEMIS (Cavallini 1998). It has certain advantages such as no "orange peel" pattern in the image which arises from tiny flatness errors of the etalons. FPI1 is a Queensgate etalon ET 100 with $50 \mathrm{~mm}$ aperture, $125 \mu$ spacing ( \pm few $\mu$ for wavelength scanning), and a finesse $\mathcal{F} \approx 40$. FPI1 has replaced the originally mounted universal birefringent filter (UBF) for further order sorting. This replacement has increased the transmission of the spectrometer by a factor of 5 , at least. The narrowband FPI2 is from Burleigh Instruments Ltd. and has an aperture of $35 \mathrm{~mm}$, variable spacing from few $100 \mu$ to several $\mathrm{mm}$, and a finesse $\mathcal{F} \approx 30$. To avoid reflexes on CCD2 arising from reflections between the two FPIs, FPI1 is slightly inclined against the optical axis. This reduces somewhat its finesse to $\mathcal{F} \approx 35$. The wavelength scanning 
is done by computer controlled changes of the spacings of the etalons by means of Piezo elements.

The broad- and narrow-band images are taken strictly simultaneously, which is important for image restoration (see below). The increase of the overall transmission of the spectrometer in replacing the UBF by FPI1 was a substantial improvement: (1) it allowed to dismount the formerly installed image amplifier in front of CCD2 and thus to increase the detective quantum efficiency; (2) the exposure times for the narrow-band images could be decreased and are now approximately $10-20 \mathrm{~ms}$ for observation without polarimeter and 20-30 ms with the $V$ polarimeter. This allows the application of speckle interferometry for image reconstruction.

Further equipment (not shown in Fig. 1, but see Koschinsky 2001) concerns a laser beam for finesse adjustment and a beam with continuum light from a halogen lamp.

\section{Observations}

The observations for the present investigation were obtained in October 1999 under very good seeing conditions (Fried parameter $r_{0}=13-14 \mathrm{~cm}$ ) with the above "Göttingen" two-dimensional spectrometer, including the $V$ polarimeter, in the VTT. Several regions were selected. On October 22, 1999, a short time series of wavelength scans of the Fe I 6302.5 $\AA$ line (Landé factor $g=2.5$ ) were taken from a very quiet region close to disc center for purposes of calibration and tests of the software for data analysis. Yet these observations show some interesting features on small-scale magnetic elements and on the limitations of the present setup (Sect. 5.1). On the same October 22 a bipolar active region (NOAA 8738) at $\cos \theta=0.97$ was observed with several wavelength scans, also in Fe I $6302.5 \AA$. It contained some pores and abnormal granulation as well as a small sunspot. A FWHM of $66 \mathrm{m \AA}$ of the spectrometer was chosen in order to reduce noise by collecting "many" photons. The exposure time was $20 \mathrm{~ms}$. We thus, by using a rather broad spectral profile compared to high resolution spectrographs and by applying short exposure times, sacrifice on spectral purity, on purpose. We aim at high spatial resolution via image restoration. For this one has to make compromises between exposure time, spectral resolution, and noise.

A spacing of $27 \mathrm{~m} \AA$ between adjacent wavelength positions was chosen for the spectral scans. With the narrowband channel 5 images at each of 28 wavelength positions were taken which gives a wavelength coverage of approximately $730 \mathrm{~m} \AA$.

One wavelength scan takes $39 \mathrm{~s}$, and further $35 \mathrm{~s}$ are needed for the storage of the image data onto hard discs. Thus, the separation of two scans amounts to $74 \mathrm{~s}$. These time scales are of concern for fast small-scale processes on the Sun. We will improve the apparatus with substantially faster CCDs and computers.

Further scans had to be taken for the data reduction: dark frames, flat field scans with defocussed telescope, and scans with the continuum source. The defocussing of the telescope for the flat fields causes a change of illumination of the spectrometer and of the detectors. We found this effect always negligible, e.g. by comparing average line profiles from the quiet Sun and from the flat fields. The defocussed images contain also the information on the noise, which is needed for the data analysis. For many other observations, flat fields are taken with moving pointing of the telescope during exposure. The difficulty and the drawback with this method are that in a rapid sequence with exposure times of 20-30 ms the image motion (from the varying pointing) must be fast such that structures on the Sun are fully smoothed.

Figure 2 demonstrates the change of Stokes $I$ and $V$ profiles when an Airy FPI transmission function of $66 \mathrm{~m} \AA$ FWHM is applied. The solar Fe I $6302.5 \AA$ line was taken from the Fourier Transform Spectrometer Atlas by Brault \& Neckel (1987). In Fig. 2b, a magnetic flux density of $0.1 \mathrm{~T}$ ( $\widehat{=} 1000$ Gauss), parallel to the line of sight and independent of height was assumed to separate the observed $I_{\lambda}$ profile into the circularly polarized components $\frac{1}{2}(I+V)_{\lambda}$ and $\frac{1}{2}(I-V)_{\lambda}$. This procedure assumes also that the magnetic and non-magnetic atmospheres possess equal thermal structure. Various fill factors for the magnetic relative to the non-magnetic area were applied.

It is seen that the $I$ and $V$ profiles change noticeably. Comparison with magnetic flux tube models or with numerical simulations of flux tube dynamics should apply the same convolution with Airy's function to the predicted profiles. In Fig. 2c we show the dependence of the Stokes $V$ amplitude (left ordinate, upper straight lines) and of the separation of the circularly polarized line profiles (right ordinate, lower curves) on the area fill factor. The amplitudes and line separations are directly proportional to the magnetic flux (or fill factor) in the resolution element (weak field limit). While the amplitudes depend on the spectral resolution, the line separations do not. At low area fill factors, in the range of $0.01-0.02$, the separations amount to $1-2 \mathrm{~m} \AA$ ( $=20$ Gauss average field strength) and their determination becomes inaccurate. In these cases, the errors compared to the expected values amount to $30 \%$.

\section{Data analysis}

\subsection{Pre-analysis}

First, certain data processings were applied, some of which are of special need for FPI spectro-polarimetric data.

Average dark frames were subtracted. Fluctuations of the sky transparency are obtained from the broad-band images. The gain tables of the CCDs were obtained from the flat field scans. They depend, for the narrow-band channel, somewhat on the wavelength position of the FPI transmission curves. Thus each wavelength setting needs its own gain table for CCD2. Yet, this dependence on the wavelength setting is small, such that flat field frames from two adjacent wavelength positions could be averaged. 

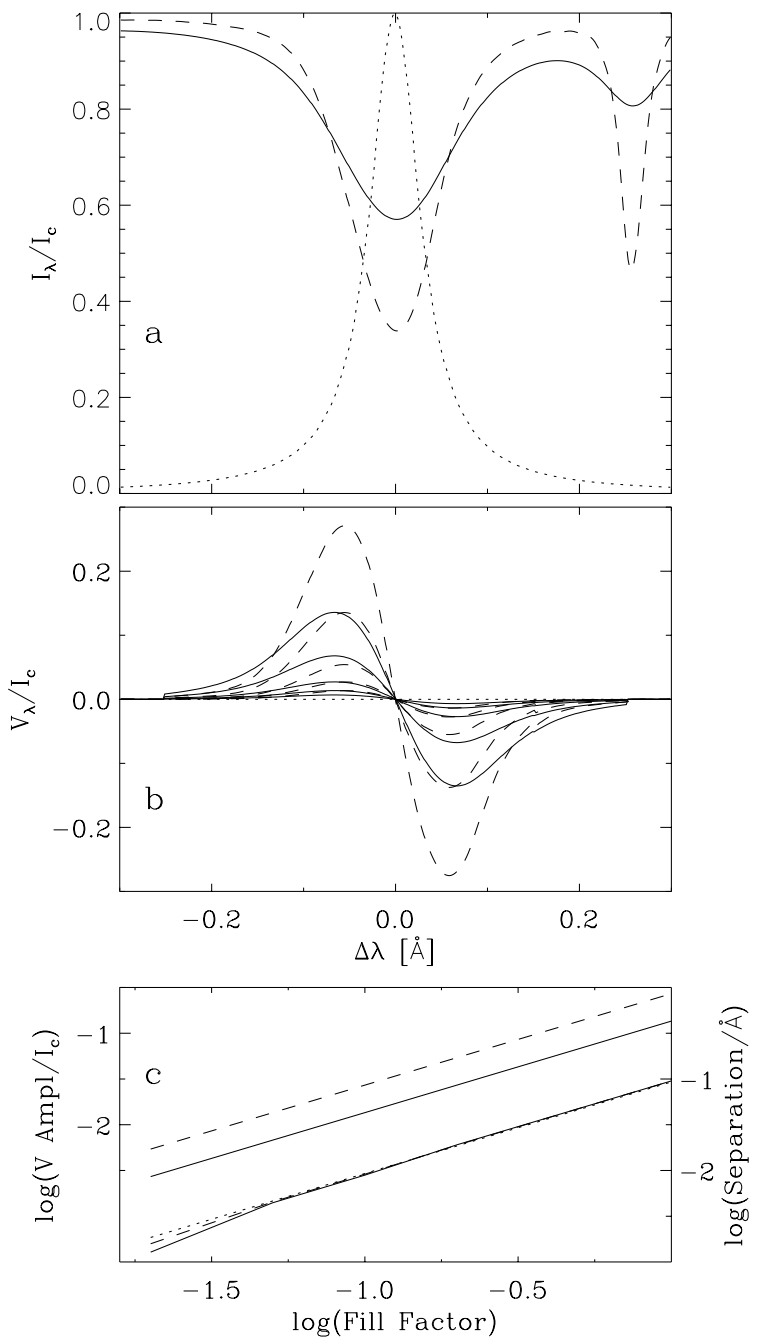

Fig. 2. Effects of broadening on Stokes $I$ and $V$ profiles of Fe I $6302.5 \AA$ by convolution with a FPI Airy function of $66 \mathrm{~m} \AA$ FWHM. a) Stokes $I$ profiles after convolution (solid) and the original profile (dashed) taken from the FTS atlas by Brault \& Neckel (1987); dotted: Airy's FPI function. b) Stokes $V$ profiles after convolution (solid) and without convolution (dashed). A constant magnetic field of $0.1 \mathrm{~T}$ along the line of sight and various area fill factors are adopted. The fill factors are, with decreasing amplitude of $V: 1.0,0.5,0.2,0.1,0.05$ (see text). c) Upper two lines: dependence of the $V$ amplitude on the fill factor for the convolved $V$ (solid) and original (dashed) profiles; lower lines: dependence of the separation of the centers of gravity of the $\frac{1}{2}(I+V)$ and $\frac{1}{2}(I-V)$ profiles on the area fill factor for the convolved (solid) and original (dashed) profiles. The dotted line gives the expected separation of the centers of gravity

This reduces (photon) noise introduced by the flat fielding. To keep the wavelength dependence of the solar spectrum during the correction processes, each gain table of CCD2 was normalized to an average value of 1 .

Since FPI1 is inclined against the optical axis the transmission of the spectrometer varies across the field of view. In addition, the two FPIs do not scan the wavelength exactly in parallel which changes slightly this dependence of transmission with wavelength. Both the dependence of transmission over the field of view and its variation with wavelength are retrieved from the flat field scans.

Next, one must be aware that the wavelengths with the highest spectrometer transmission vary also across the field of view, due to the mounting of the FPIs in the parallel beam.

Image motion was taken out by correlating the broadband images. This reduces the fields of view by few arcseconds, depending on seeing. The same corrections as determined from the broad-band images apply to the narrow-band images. Small displacements of the narrowband against broad-band images due to not exact alignment and thermal drifts are measured again from correlations between narrow-band images in the continuum spectral range and the broad-band images.

Furthermore, for image reconstruction with speckle methods, i.e. removal of seeing effects within isoplanatic patches, the frames were divided into partly overlapping sub-fields of $64 \times 64$ pixel each ( $\left.\widehat{=} 6{ }^{\prime \prime} 4 \times 66^{\prime \prime} 4\right)$. At the end, these sub-fields were recombined again to full images.

\subsection{Speckle reconstruction}

Broad-band images. While the two-dimensional spectrometer scans over a Fraunhofer line, CCD1 takes 140 images in integrated light. These are used for image restoration with well established speckle methods (e.g. de Boer et al. 1992; de Boer 1996; Wilken et al. 1997). The spectral ratio method (von der Lühe 1984) gives estimates of the amplitude correction factors (in Fourier space). During this processing, the seeing parameter $\alpha=r_{0} / D$ $\left(r_{0}=\right.$ Fried parameter, $D=$ telescope aperture $\left.=70 \mathrm{~cm}\right)$ is calculated. The (Fourier) phases are reliably obtained with the speckle masking method (Weigelt 1977; Weigelt \& Wirnitzer 1983; Lohmann et al. 1983).

Narrow-band images. The spectrometric images, i.e. sub-images, are reconstructed following Keller \& von der Lühe (1992) and as described by Krieg et al. (1999). In principle, given the reconstructed, "true" broad-band object $\hat{O}_{\mathrm{b}}$ (in Fourier space, with $\hat{\text {. denot- }}$ ing estimates) and the broad-band images $I_{\mathrm{b}, l}$ observed at time $l$, one can retrieve the instantaneous optical transfer functions $S_{l}$, which hold also for the simultaneously taken narrow-band images $I_{\mathrm{n}, l}$. After re-ordering and summation over the 5 images taken at each wavelength position (see Krieg et al. 1999) one obtains an estimate of the narrowband object $\hat{O}_{\mathrm{n}}$

$\hat{O}_{\mathrm{n}}=H \frac{\sum_{l} I_{\mathrm{n}, l} I_{\mathrm{b}, l}^{*}}{\sum_{l}\left|I_{\mathrm{b}, l}\right|^{2}} \hat{O}_{\mathrm{b}}$.

Here, a (real) noise filter $H$ is introduced. We have tested optimum filters (Brault \& White 1971; Löfdahl 1996; Krieg et al. 1999). With some experience we found it easier and without noticeable change of the results to implement filters which are equal to 1 up to a certain horizontal wavenumber (independent of azimuth) and then drop quickly to 0 . In the results of the small-scale polarimetry 
presented below, this cutoff wavenumber corresponds to typically 0 .' 4 , which is thus the spatial resolution limit of our reconstructed small-band images near line center.

Certainly, the quality of the reconstructed images, i.e. the spatial resolution, depends on the instantaneous seeing conditions prevailing during the exposures at each wavelength position. To eliminate such effects one could apply the filters from the worst seeing conditions to all spectral positions. This, however would suppress the information obtained from the observations with the best seeing. A better choice is to smooth the $V_{\lambda}$ profiles along wavelength if needed.

\subsection{Instrumental polarization}

For polarimetry one has to consider instrumental polarization. We are mainly interested in the Stokes $V$ component. After passing through the telescope and the spectropolarimeter it is changed to

$V_{\lambda}^{\prime}=M_{41} I_{\lambda}+M_{42} Q_{\lambda}+M_{43} U_{\lambda}+M_{44} V_{\lambda}$,

where $M_{41} \ldots M_{44}$ are the elements in the last row of the Mueller matrix.

For small-scale magnetic elements the Stokes $Q$ and $U$ components are small, at the $10^{-3}$ level of the continuum intensity $I_{\mathrm{c}}$, even close to the solar limb. This is due to the partly cancellation of linearly polarized signals from expanding flux tubes and to the squared dependence of the $Q$ and $U$ profiles on the magnetic flux density (cf. Landi degl'Innocenti 1992; Sigwarth et al. 1999). We thus can neglect the $(Q / U) \rightarrow V$ cross talk. (This is no longer correct, of course, when performing polarimetry of regions with strong magnetic fields perpendicular to the line of sight and with large fill factors as for instance in sunspot penumbrae.)

After Capitani et al. (1989) the element $M_{44}$ is typically of the order 0.9 for coelostat systems as the VTT. We do not correct for it, since we have no better measure. It remains to determine the $I \rightarrow V$ crosstalk, i.e. the element $M_{41}$. There should be no true solar $V$ component from non-magnetic areas on the Sun. Then, Eq. (2) reads

$V_{\lambda}^{\prime}=M_{41} I_{\lambda}$

$M_{41}$ can be determined from the flat field and the quiet Sun observations. Taking averages over sub-areas of $7^{\prime \prime} \times 10^{\prime \prime}$ we obtained offsets in the range $V_{\lambda}^{\prime}=$ $(0.4 \ldots 1.0) 10^{-2} I_{\mathrm{c}}$ and a "noise" amplitude of $\delta \hat{V} \approx$ $0.510^{-2} I_{\mathrm{c}}$ (peak to peak). Both the offsets and the noise pattern persisted from scan to scan but varied from area to area within the field of view. Presumably, they result from the difference of the optical path, including the different detector areas, of the two circularly polarized beams in the apparatus and from interferences. We can reduce these effects by subtracting the offsets and the fixed pattern noise, similarly as in the second-order flat fielding introduced by Sánchez Almeida \& Martínez Pillet (1994). We then arrive at a lower detection limit, dominated by photon noise, for the $V$ amplitude of $10^{-2} I_{\mathrm{C}}$ in a $3 \times 3$ pixel area $\left(\widehat{\approx} 220 \times 220 \mathrm{~km}^{2}\right)$. This smoothing over $3 \times 3$ pixels lowers the noise, but still preserves the spatial resolution. The Stokes $V$ profiles presented below have been treated with such an area averaging.

On the other hand, we found no wavelength dependent $V_{\lambda}^{\prime}$, expected from Eq. (3), above the detection limit of approximately $210^{-3} I_{\mathrm{c}}$ in the spatially averaged quiet Sun observations (outside obvious magnetic structures). We conclude that the $I \rightarrow V$ cross talk $M_{41}$ is below this value in the present observations.

The $Q / U / V \rightarrow I$ cross talk can be of concern again when dealing with strong magnetic fields and large filling factors as in sunspots. The spectral lines $I_{\lambda}$ can then become contaminated with asymmetry caused by the crosstalk. Below, we shall therefore refrain from discussing such asymmetries observed in sunspots.

As mentioned, the mounting of the FPIs in the parallel beams has the following effect: the wavelength for which the FPIs have highest transmittance depends on the position within the field of view. This means that the solar spectrum is shifted from one point to the other. Since our $V$ polarimeter separates the beams for $\frac{1}{2}(I+V)$ and $\frac{1}{2}(I-V)$ they can be considered as stemming from two separated fields of view. In consequence, the spectra of corresponding image points in $\frac{1}{2}(I+V)_{\lambda}$ and $\frac{1}{2}(I-V)_{\lambda}$ are shifted against each other. This has been corrected for by carefully re-shifting the spectra by means of the profiles in the flat field and quiet Sun observations.

\subsection{Magnetic flux}

Observed Stokes $I$ and $V$ profiles can be used for comparison with model predictions, i.e. from numerical simulations of flux tube structure and dynamics. And one may apply inversion techniques to obtain physical parameters such as the runs of temperature, velocity, and magnetic field strength with height in the atmosphere (e.g. Bellot Rubio et al. 1997). We shall present $V_{\lambda}$ measurements which may be used for such purposes in cases where the signal to noise ratio is sufficiently large. (The corresponding $I_{\lambda}$ profiles will not be shown.)

A fast method to obtain maps of magnetic flux, that is of its component along the line of sight, is the center of gravity (COG) method by Semel (1967) and Rees \& Semel (1979). This measures simply the wavelength separation of the COGs of the $\frac{1}{2}(I+V)_{\lambda}$ and $\frac{1}{2}(I-V)_{\lambda}$ profiles. Obviously and as seen from Fig. 2c, the separation is not dependent on the spectral resolution. In addition, the $\mathrm{COG}$ method gives fluxes in each pixel for the average performance of image reconstruction, i.e. for the average seeing conditions during a spectral scan. The best images from one scan bring the wavelength separation toward the direction of best spatial resolution. There is thus no need to reduce, by filtering, all images to the lowest resolution. 


\section{Results and discussion}

The data analysis has given us a wealth of information on small-scale magnetic structures of the Sun. Here, we can present only part of the results. A broader discussion of, e.g., profile asymmetries and relationships between intensity, velocity, and magnetic fields at high spatial resolution is prepared for a forthcoming contribution.

\subsection{Quiet region near disc center}

Figure 3 shows four maps obtained from the quiet Sun observations: the speckle reconstructed integrated light image, the line of sight component of the magnetic flux in the $0^{\prime \prime} 1 \times 0^{\prime \prime} .1$ pixels, the continuum intensity, and the line center intensity. The rms fluctuation of the magnetic flux is at approximately $0.2210^{8} \mathrm{~Wb}$. We thus adopt a detection limit of $|\Phi|=0.510^{8} \mathrm{~Wb}(\widehat{=} 2 \sigma)$.

The areas marked by rectangles in Fig. 3 contain the most significant magnetic flux. We notice a bow of magnetic features from the area close to the right border towards upper left. It is also seen in the line center image and remained visible during the $5 \mathrm{~min}$ of this short time series. Presumably, it represents weak network. Apart from that, the field of view appears very non-active, as is also seen in the magnetogram of this day from NSO at Kitt Peak. The strongest flux in the field of view corresponds to a magnetic fieldstrength, averaged over one pixel, of $310^{-2} \mathrm{~T}$.

The maximum of the flux (per pixel) of the magnetic element at the lower left corner in Fig. 3 strengthened during the $5 \mathrm{~min}$ sequence by a factor of 1.5 . Its counterpart in the integrated light image is a photospheric bright point which was "compressed" by nearby growing granules.

We present in Fig. 4 again the magnetogram and the speckle reconstructed image of this sub-area and in addition its velocity map. The latter was obtained from the COG of the Stokes I profile. We adopted the COG position averaged over the total field of view as zero velocity. The velocities contain both granular and oscillatory motions. Future analysis should disentangle the two contributions via filtering of time sequences longer than the one used here (cf. Espagnet et al. 1995). The magnetic structure is elongated and consists possibly of two flux tubes, or two bundles of flux tubes. It is located at a strong intergranular downdraft. In fact, the fastest downflow of $1.2 \mathrm{~km} \mathrm{~s}^{-1}$ in this sub-field occured close to the magnetic structure, but not exactly in it. From the position of the zero crossing of the $V$ profile, which gives the velocity of the magnetized plasma, one obtains a downflow of $1.5 \pm 0.5 \mathrm{~km} \mathrm{~s}^{-1}$. Possibly, one witnesses here in a snapshot a convective intensification of flux tubes as, e.g., demonstrated with numerical simulations by Steiner et al. (1998) and Grossmann-Doerth et al. (1998).

The flux region at the right border of Fig. 3 shows substructure, and we use this area for the discussion of temporal evolution. Figure 5 shows a short time sequence of it together with its "non-magnetic" surroundings. The maps are separated in time by $74 \mathrm{~s}$, start is at the top.

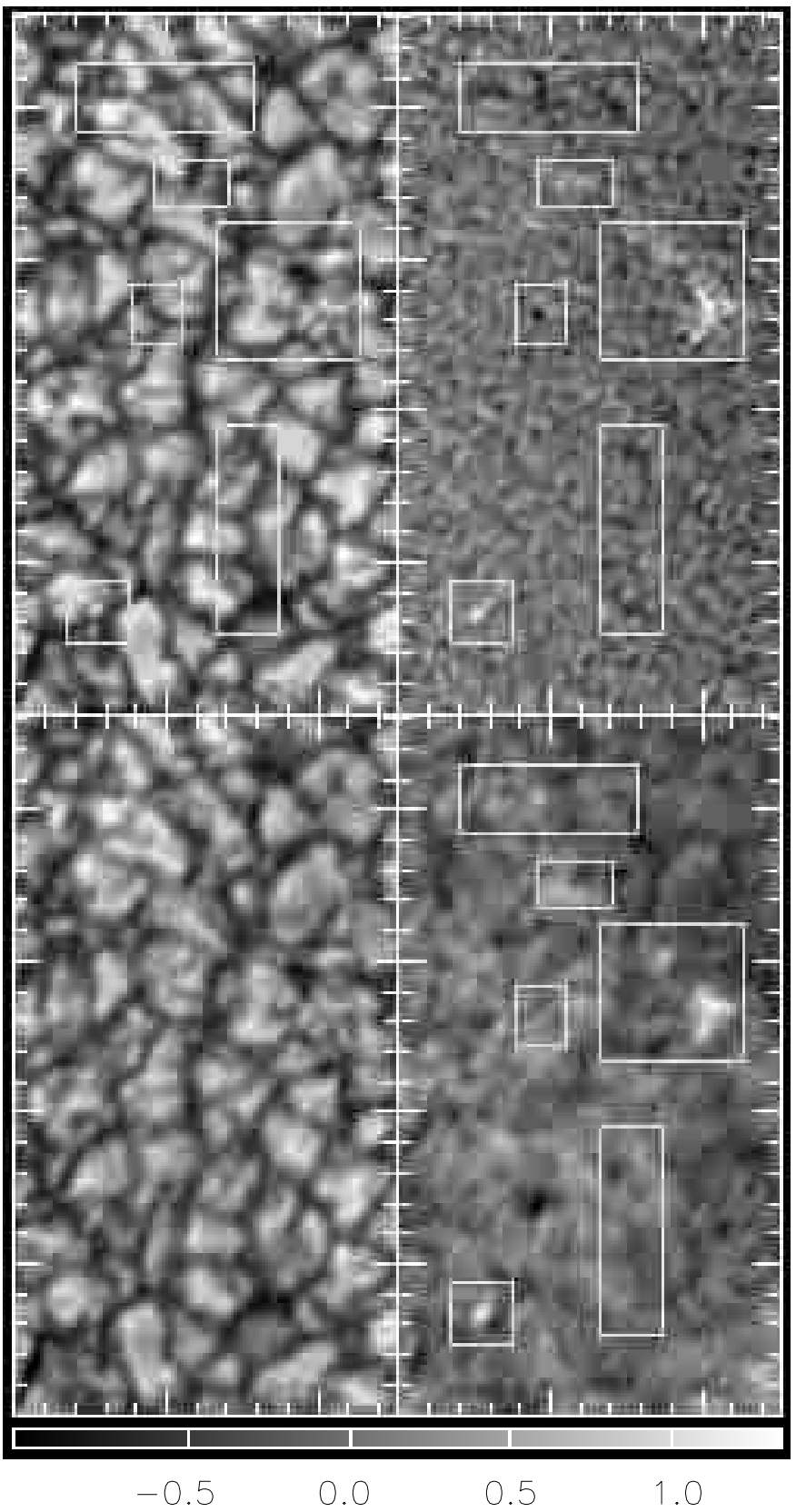

Fig. 3. Maps of a quiet Sun disc center area: speckle reconstructed integrated light image (upper left), magnetic flux along the line of sight from COG (upper right), continuum image from the narrow-band channel (lower left), and line center intensity (lower right). The tickmarks are at $1^{\prime \prime}$ distance, the field of view is 12 ." $5 \times 23^{\prime \prime} .1$. The bar at the bottom gives the scaling of the magnetic flux per pixel $\left(00^{\prime \prime} 1 \times 0^{\prime \prime} .1\right)$ in $10^{8} \mathrm{~Wb}$ for the magnetic field map

The left images are the speckle reconstructions from integrated light, the right images represent the magnetic flux. The images are overlaid with Stokes $V$ profiles centered at the pixels from which they were obtained.

The maximum $V$ amplitudes amount to $0.025 I_{\mathrm{c}}$. By means of Fig. 2, we can approximately correct for the limited spectral resolution of the FPI setup and estimate maximum amplitudes of $0.05 I_{\mathrm{c}}$. 


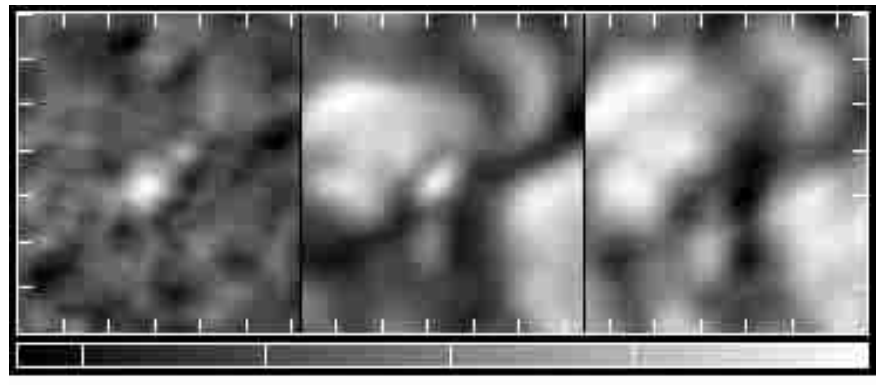

Fig. 4. Magnetic map (left), speckle reconstructed integrated light image (middle), and velocity from the COG method (right) of the magnetic flux region at the lower left corner of Fig. 3; bar at bottom: velocity in $\mathrm{km} \mathrm{s}^{-1}$ with bright indicating upward flow. Tickmarks are at 0.5 distance

We must interpret Fig. 5 with caution, since the spatial resolution of the magnetograms is 0 ". 4 , at best, and their noise poses a lower detection limit for the flux. The positive magnetic flux (magnetic field towards observer) is mostly located above small-scale "white light" features. These are the three bright "points" to the right of the exploding granule and evolving from the second time step on. However, positive flux is also detected above areas which are inconspicuous in white light or even clearly darker than average. This is seen e.g. from the $V$ profiles marked by rectangles in the third time step. Just to the left of these two positions, at the border of the exploding granule and just at the detection limit, negative flux becomes visible. Possibly, convective re-shuffling of magnetic flux is at work. The whole evolution appears dominated by the dynamics of the exploding granule. A sort of bow of the magnetic structure develops by the intrusion of the granular material. The latter itself does not carry detectable flux. Roudier et al. (1994) have drawn similar conclusions from the temporal evolution of photospheric bright points.

\subsection{Active region: Pores and abnormal granulation}

We present in Fig. 6 a speckle reconstructed, integrated light image (left) together with its magnetogram of an active region with small, not fully developed pores. The images are again overlaid with $V$ profiles, which are well defined this time. The maximum measured $V$ amplitudes outside pores amount to $0.08 I_{\mathrm{c}}$. We may again correct this value for the limited spectral resolution by means of Fig. 2. One thus obtains $V$ amplitudes of $0.16 I_{\mathrm{c}}$.

Most of the magnetic flux in this area is negative, i.e. the field is directed away from the observer. The maximum field strength from the COG method is found in the pore located in the central upper part of the field of view and amounts to $0.08 \mathrm{~T}$. This is substantially smaller than the value of approximately $0.2 \mathrm{~T}$ given by e.g. Sütterlin (1996, Fig. 23, 1998). The reason for the low value found here comes likely from the scattered light produced in the Earth's atmosphere and in the telescope. We took not special care of it for these observations. False light from

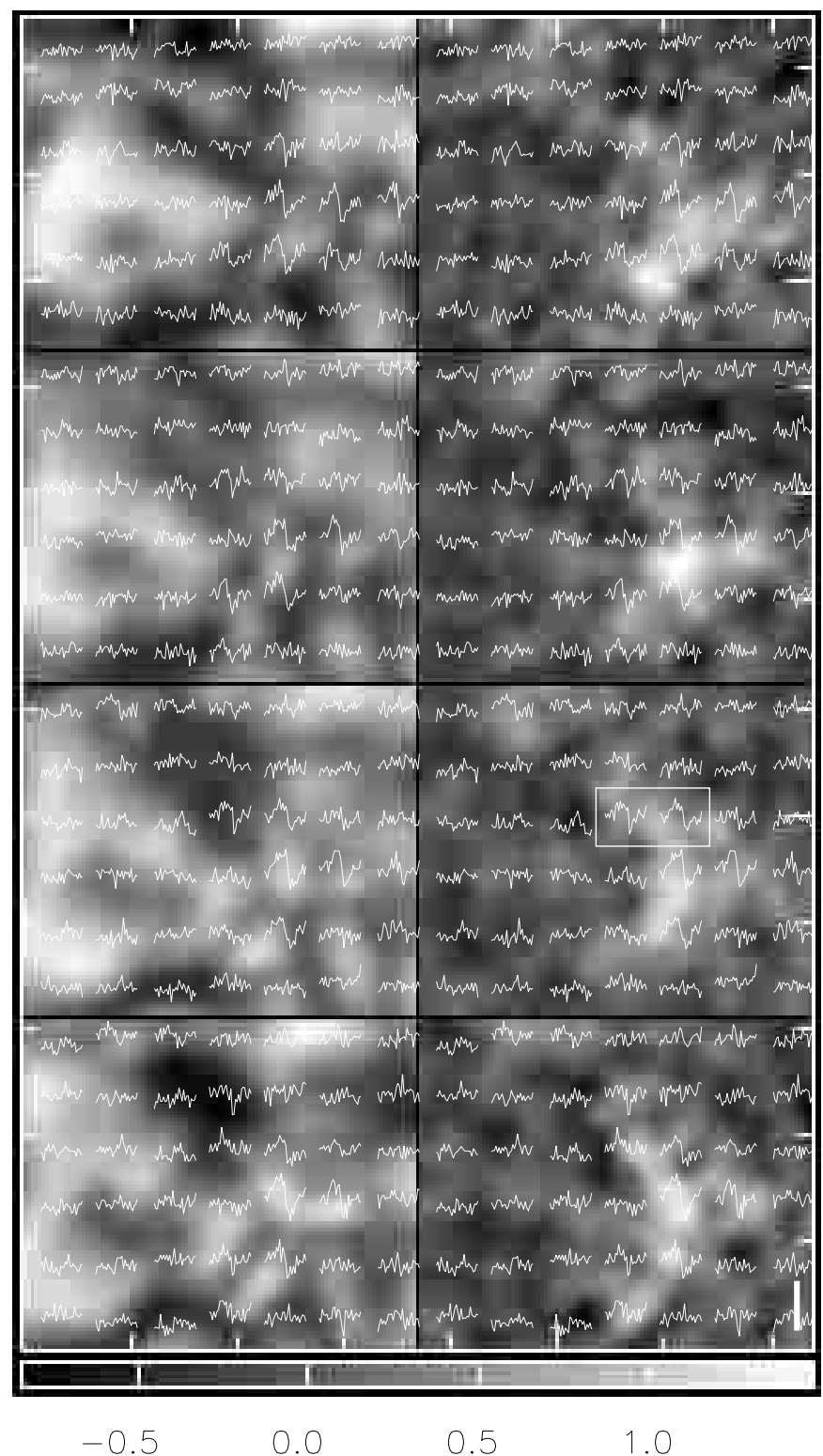

Fig. 5. Time series (top to bottom, separation of $74 \mathrm{~s}$ ) of magnetic flux evolution (region at right border of Fig. 3). Left column: speckle reconstructed images; right column: flux maps; bar at bottom: magnetic flux (in one pixel), unit $10^{8} \mathrm{~Wb}$. The images are overlaid with the Stokes $V$ profiles centered at the pixels from which they were obtained. The length of the vertical bar at the lower right corner indicates a $V$ amplitude of $0.05 I_{\mathrm{c}}$. Tickmarks are at $1^{\prime \prime}$ distance. See the text for the rectangles

outside the pores introduces an unshifted profile and thus can largely reduce the field strength measured with the COG method. We tentatively adopt that, with a fill factor of 1 and for strong fields of $0.2 \mathrm{~T}$ the amplitude of the Stokes $V$ profile of the Fe I 6302 line is saturated and that therefore the separation of the $V$ extrema gives the field strength. (A Zeeman splitting of the $I$ profile is hardly detectable and not reliable in view of the spectral resolution of $66 \mathrm{~mA}$.) We then arrive at a maximum of $0.21 \mathrm{~T}$ in the largest pore in the upper part of Fig. 6. We have 


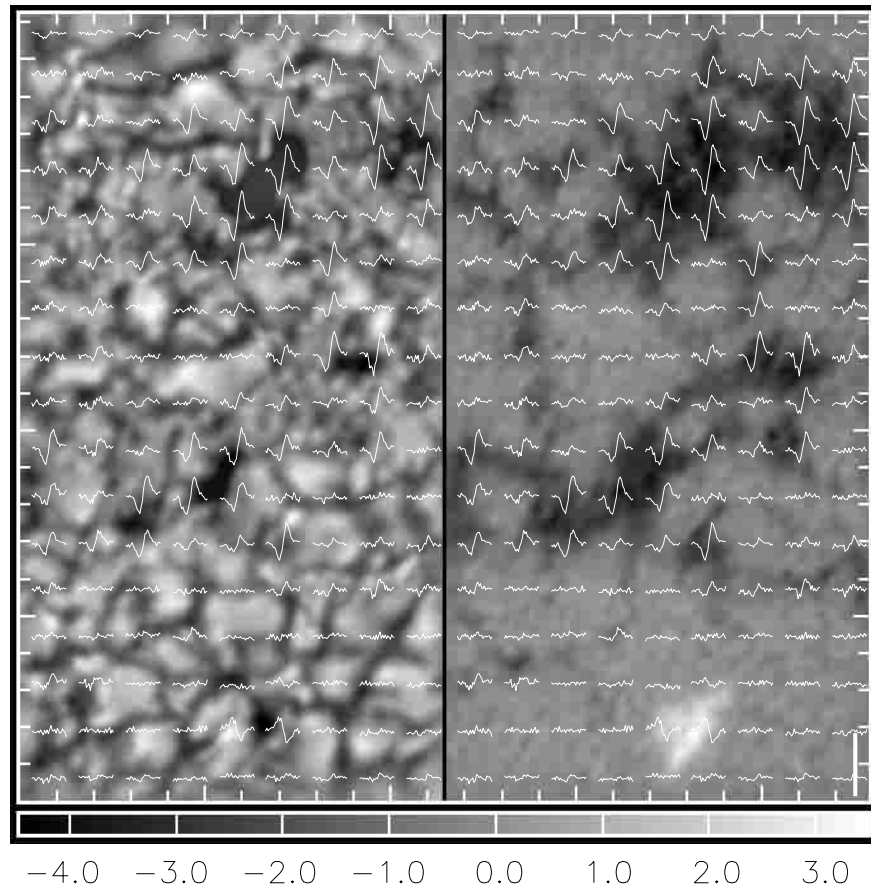

Fig. 6. Speckle reconstructed image (left) and magnetic flux map (right, from COG) of active region near disc center. The images are overlaid with the Stokes $V$ profiles centered at the pixels from which they were obtained. The length of the vertical bar at the lower right corner indicates a $V$ amplitude of $0.2 I_{\mathrm{c}}$. Tickmarks are at $1^{\prime \prime}$ distance. Bar at bottom: scaling of magnetic flux in $10^{8} \mathrm{~Wb}$

tested such a magnetic field measurement using $0.2 \mathrm{~T}$, instead of $0.1 \mathrm{~T}$, in the model for Fig. 2. The separation of the $V$ extrema returns the field strength within $2.5 \%$ accuracy. The convolution of the $V$ profiles with the profile of the spectrometer (Airy's function) adds an error of only $3 \%$. A further justification for this measurement of field strength comes from model calculations by Amer \& Kneer (1993) of the FeI $6173.3 \AA$ line which has the same Landé factor $g=2.5$ as the $6302.5 \AA$ line, although it is somewhat weaker than the latter. At magnetic field strengths $\geq 0.15 \mathrm{~T}$ the extrema of the $V$ profile do not increase any more with field strength, but separate.

Much of the field of view of Fig. 6 is covered with abnormal granulation which contains magnetic flux. It is now important to know whether the flux is located in the bright abnormal granulation features or in the darker spaces in between them. Likewise, the relation between magnetic flux and line center intensity, i.e. essentially the temperature structure in medium to high photospheric layers, is of interest. Figure 7, then, gives scatter plots of magnetic flux vs. granulation intensity and vs. line center intensity. For these, only areas of abnormal granulation were selected, data from pores are not included.

Firstly, we notice that there exists no detectable correlation between magnetic flux and abnormal granular intensity, although the magnetogram in Fig. 6 does show structure. There may be several reasons for this negative

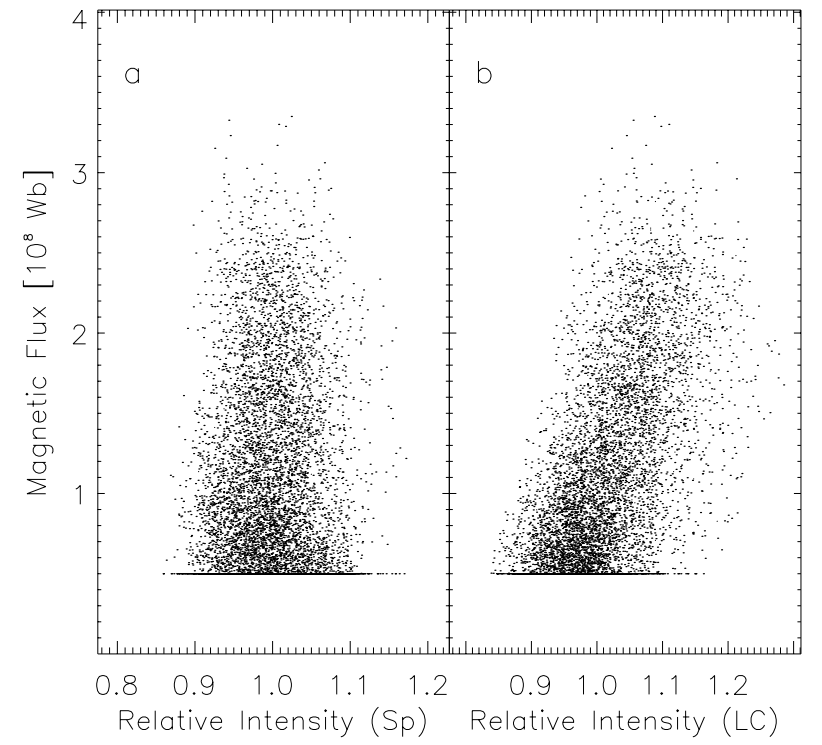

Fig. 7. a) Magnetic flux (in each pixel) vs. white light intensity in selected areas with anomalous granulation (SP on the abscissa indicates speckle reconstructed image). b) Magnetic flux vs. line center intensity (LC on the abscissa). The intensities are relative to the mean intensities of the selected areas. The lower detection limit for flux is $0.510^{8} \mathrm{~Wb}$

\section{finding:}

(1) The magnetic field is located in both bright "granules" and darker spaces in between the granules and is essentially equally strong in both features;

(2) The magnetic field is located preferentially in either bright or dark features but has merged at atmospheric heights from which the signal for the magnetic flux is obtained;

(3) Close inspection of some areas with small bright points shows that they contain substantially less magnetic flux than expected for abnormal granulation. Thus, small-scale bright features, or conglomorates of them, in white light pictures do often, but not necessarily indicate magnetic flux. It was already noted by Keller \& von der Lühe (1992) that small-scale magnetic flux elements do not correlate with small-scale brightenings in integrated light;

(4) The spatial resolution is not yet sufficient to find exactly the locations of magnetic flux.

Observations with still higher spatial resolution and with magnetically sensitive lines formed at low atmospheric heights are needed to come to a clearer conclusion.

Secondly, the line center intensities are well correlated with the magnetic flux, as known from many investigations. The stronger the flux the higher is the temperature in high atmospheric layers.

A word on the $V$ asymmetry appears appropriate. Close inspection of the $V$ profiles in Fig. 6 shows that these are sometimes strongly asymmetric and occasionally exhibit two lobes on one side of the wavelength axis. This is already seen in the magnetic flux features of the quiet Sun, Fig. 5. $Q / U \rightarrow V$ cross talk is unlikely to produce such abnormal $V$ profiles, although we cannot fully exclude it. The amplitudes of the $Q$ and $U$ profiles are 
usually found to be an order of magnitude smaller than those of the $V$ profiles, especially near disc center (e.g. Sigwarth et al. 1999; Sánchez Almeida \& Lites 2000).

Sánchez Almeida (1997) and Sánchez Almeida \& Lites (2000) have interpreted the abnormal $V$ profiles with the MISMA hypothesis (MIcro-Structured Magnetic Atmosphere). MISMAs contain flows and bundles of optically thin flux tubes with $10 \mathrm{~km}$ diameter, or less, and with both polarities. Their substructure cannot be resolved, in principle. Grossmann-Doerth et al. (2000) can explain the anomalies by special flow situations in magnetic canopies. However, the $V$ amplitudes observed in these latter works are well below $10^{-2} I_{\mathrm{c}}$, below our detection limit. Possibly, the high spatial resolution achieved here allows to isolate such dynamic flux tubes, or bundles of flux tubes, with large fill factors. Yet further work on the analysis of the present data (classification, smoothing, averaging, determination of cross talk) is needed. This will be pursued in a further investigation.

\subsection{Active region: Small sunspot}

Figure 8, finally, gives images from the small sunspot in the same active region as the pores and the abnormal granulation above. We show the speckle reconstruction of the integrated light observations, the magnetogram, the continuum image and the line center image. The maximum magnetic field strength in the umbra was $0.09 \mathrm{~T}$, again a very low value for sunspots. And again, the reason for this low value is in the false light which reduces the field strength as measured with the COG method. As above, for the field strength in the pores, we obtain from the separation of the extrema of the $V$ profile a maximum field strength of $0.233 \mathrm{~T}$.

It is noteworthy that the surrounding of the sunspot contains flux only at very few places, with preferentially the same polarity. Most of the ambient quiet area does not posses detectable flux. The granular pattern is normal, and the line center image does not exhibit brightenings, in most parts, to be related with magnetic fields. Returned magnetic flux elements, i.e. with polarity opposite to that of the sunspot, are not conspicuous.

Most of the outer penumbral magnetic filaments in the magnetogram are related to dark filaments. There, the flux component towards the observer seems to be larger than in the bright penumbral points. But, upon close inspection, one can also find few bright filaments with enhanced flux. However, as mentioned above (4.3, instrumental polarization), the measurements of polarized light from a penumbra have to be interpreted with caution due to the possible $Q / U \rightarrow V$ instrumental cross talk. A further discussion of the sunspot results has thus to await measurements of this cross talk. Also, the velocities in the penumbral filaments will be of interest. Such an investigation of the penumbra dynamics at small scales, as in our data, is beyond the scope of this paper, and we refer to the review by Wiehr (1999, see also many references therein).

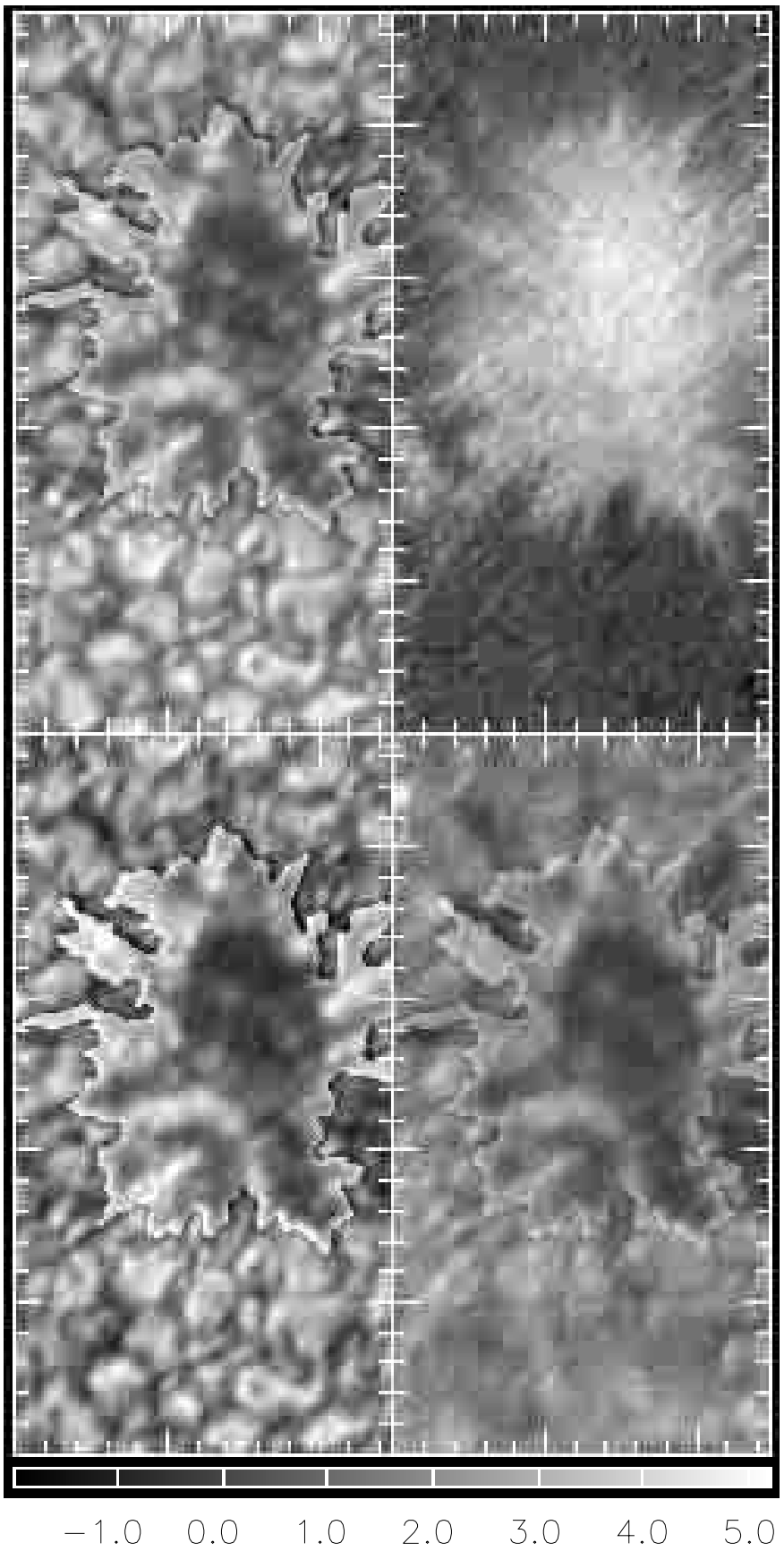

Fig. 8. Speckle reconstructed image (upper left), magnetic flux map (upper right, from COG), continuum intensity (lower left), and line center intensity (lower right) of small sunspot near disc center. Tickmarks are at $1^{\prime \prime}$ distance. Bar at bottom: scaling of magnetic flux in $10^{8} \mathrm{~Wb}$. The intensities of the sunspot were enhanced to show its internal small-scale structure

\section{Conclusion}

We have demonstrated the high potential of twodimensional spectro-polarimetry in combination with methods of speckle reconstruction. The computational load for image reduction is large, however not unsurmountable with today's and future computer facilities. With these methods of observation and data treatment, it was possible to arrive at high spatial resolution and to 
enter the realms of small-scale magnetic field dynamics by direct observation. The results presented above can be compared with polarized radiation expected from numerical models, of course only after adequate smoothing of the simulated data to the spatial and spectral resolution of the observations.

The work and the results above represent a further step towards the physics of solar finestructure. The next steps will be to observe longer time sequences with higher spatial and spectral resolution. The instrumental improvements for this purpose (faster computers, narrower bandwidths) are underway. Modern detector technology will also allow faster cadences of exposures during wavelength scans, thus will allow to take more images per wavelength scan and to reduce the photon noise.

To arrive at substantially higher spatial and spectral resolution than achieved here telescopes with larger aperture than the VTT are needed. There exist well developed plans for a $1.5 \mathrm{~m}$ Gregorian solar telescope at the Observatorio del Teide. This will possibly give, at higher spatial resolution than the VTT, larger fill factors, thus stronger signals at small scales. A Gregorian telescope is also much less sensitive to instrumental cross talk of the components of the Stokes vector than a coelostat system as the VTT. Further improvement could come from a polarimetric device like the Zurich Imaging Polarimeter (ZIMPOL, Keller et al. 1992). This has the advantage of using exactly the same optical paths and detector areas for the various polarized light beams, which leads to high polarimetric accuracy. Finally, in the near future, one will take profit from Adaptive Optics. Even then, image reconstruction will still be necessary since Adaptive Optics should not be expected to fully correct for the seeing effects in large fields of view. But the reconstruction process will certainly be much eased with high quality input data.

Acknowledgements. Financial support by the Deutsche Forschungsgemeinschaft through grants KN 152/19-1,2 is gratefully acknowledged. J. H. acknowledges the financial support of the Austrian Fonds zur Förderung der wissenschaftlichen Forschung (Erwin-Schrödinger-Stipendium $J$-1802 PHY) and thanks for the hospitality and the support of the Universitäts-Sternwarte Göttingen. The Vacuum Tower Telescope at the Spanish Observatorio del Teide of the Instituto de Astrofísica de Canarias/Tenerife is operated by the Kiepenheuer-Institut für Sonnenphysik in Freiburg, Germany.

\section{References}

Amer, M. A., \& Kneer, F. 1993, A\&A, 273, 304

Beckers, J. M., \& Schröter, E. H. 1968, Solar Phys., 4, 142

Bellot Rubio, L. R., Ruiz Cobo, B., \& Collados, M. 1997, ApJ, 478, L45

Bendlin, C. 1993, Ph.D. Thesis, Göttingen University

Bendlin, C., \& Volkmer, R. 1995, A\&AS, 112, 371

Bendlin, C., Volkmer, R., \& Kneer, F. 1992, A\&A, 257, 817

Brault, J. W., \& Neckel, H. 1987, Spectral Atlas of Solar Absolute Disk-averaged and Disk-center Intensities from 3290 to $12510 \AA$, unpublished
Brault, J. W., \& White, R. O. 1971, A\&A, 13, 169

Capitani, C., Cavallini, F., Ceppatelli, G., et al. 1989, Solar Phys., 120, 173

Cavallini, F. 1998, A\&AS, 128, 589

de Boer, C. R. 1996, A\&AS, 120, 195

de Boer, C. R., Kneer, F., \& Nesis, A. 1992, A\&A, 257, L4

Espagnet, O., Muller, R., Roudier, Th., Mein, N., \& Mein, P. 1995, A\&AS, 109, 79

Grossmann-Doerth, U., Schüssler, M., \& Steiner, O. 1998, A\&A, 337, 928

Grossmann-Doerth, U., Schüssler, M., Sigwarth, M., \& Steiner, O. 2000, A\&A, 357, 351

Keller, C. U., \& von der Lühe, O. 1992, A\&A, 261, 321

Keller, C. U., Aebersold, F., Egger, U., et al. 1992, Zürich Imaging Stokes Polarimeter ZIMPOL I - Design Review, LEST Technical Report Series No. 53, ed. O. Engvold, \& $\varnothing$. Hauge, Oslo University

Kentischer, T., Schmidt, W., Sigwarth, M., \& von Uexküll, M. 1998, A\&A, 340, 569

Koschinsky, M. 2001, Ph.D. Thesis, Göttingen University

Krieg, J., Kneer, F., Koschinsky, M., Ritter, C., \& Starck, J.-L. 1998, Ground-based observation with high spatial and spectral resolution, in A Crossroads for European Solar and Heliospheric Physics, ed. R. H. Harris, F. Moreno Insertis, \& E. Priest, ESA SP-417, 317

Krieg, J., Wunnenberg, M., Kneer, F., Koschinsky, M., \& Ritter, C. 1999, A\&A, 343, 983

Landi degl'Innocenti, E. 1992, in Magnetic Field Measurements, ed. F. Sánchez, M. Collados, \& M. Vázquez, Solar observations: Techniques and interpretation (Cambridge: Cambridge University Press), 73

Löfdahl, M. G. 1996, Ph.D. Thesis, Stockholm University

Lohmann, A. W., Weigelt, G. P., \& Wirnitzer, B. 1983, Appl. Opt., 22, 4028

Rees, D. E., \& Semel, M. D. 1979, A\&A, 74, 1

Roudier, T., Espagnet, O., Muller, R., \& Vigneaux, J. 1994, A\&A, 287, 982

Sánchez Almeida, J. 1997, ApJ, 491, 993

Sánchez Almeida, J., \& Lites, B. W. 2000, ApJ, 532, 1215

Sánchez Almeida, J., \& Martínez Pillet, V. 1994, ApJ, 424, 1014

Semel, M. D. 1967, Ann. Astrophys., 30, 513

Sigwarth, M., Balasubramaniam, K. S., Knölker, M., \& Schmidt, W. 1999, ApJ, 349, 941

Steiner, O., Grossmann-Doerth, U., Knölker, M., \& Schüssler, M. 1998, ApJ, 495, 468

Stenflo, J. O. 1973, Solar Phys., 32, 41

Stenflo, J. O. 1994, Solar Magnetic Fields - Polarized Radiation Diagnostics (Kluwer Academic Publ.), 273

Stolpe, F., \& Kneer, F. 2000, A\&A, 353, 1094

Sütterlin, P. 1996, Ph.D. Thesis, Freiburg University

Sütterlin, P. 1998, A\&A, 333, 305

Volkmer, R. 1995, Ph.D. Thesis, Göttingen University

Volkmer, R., Kneer, F., \& Bendlin, C. 1995, A\&A, 304, L1

von der Lühe, O. 1984, J. Opt. Soc. Am., A1, 510

Weigelt, G. P. 1977, Opt. Comm., 21, 55

Weigelt, G. P., \& Wirnitzer, B. 1983, Opt. Lett., 8, 389

Wiehr, E. 1999, Mass Motions and Magnetic Fields in Penumbrae, in Third Advances in Solar Physics Euroconference: Magnetic Fields and Oscillations, ed. B. Schmieder, A. Hofmann, \& J. Staude, Astron. Soc. Pac. Conf. Ser., 184, 86

Wilken, V., de Boer, C. R. Denker, C., \& Kneer, F. 1997, A\&A, 325,819 